\title{
A memory management unit and cache controller for the MARS system
}

\author{
Feipei Lai, Chyuan-Yow Wu, and Tai-Ming Parng \\ Department of Electrical Engineering \\ $\&$ \\ Department of Computer Science \\ National Taiwan University \\ Taipei, Taiwan, R.O.C.
}

\begin{abstract}
For large caches, the interaction between cache access and address translation affects the machine cycle time and the access time to memory. The physically addressed caches slow down the cache access due to the virtual address translation. The virtually addressed caches is faster, but the synonym problem is difficult to handle. By some software constraints and hardware support, our virtually addressed physically tagged caches can achieve the same speed as traditional virtually addressed cache and solve the synonym problem. The design of delayed miss signal makes the TLB access depart from the critical path of the cache access. A simple method to solve the TLB coherence is implemented in this chip and only a little hardware is required.
\end{abstract}

\section{Introduction}

Due to the temporal and spatial locality of program, the cache memories have been proven to be effective in reducing the memory latency for most uniprocessors [1]. For bus-based, shared memory multiprocessors, the performance is bounded by the saturation of the bus. Caches are also investigated in order to find some way to reduce the bus traffic [2].

Most caches are set-associative. They map an address into a set of entries in the cache and search associatively within the set to find the desired word. The set size (the degree of associativity) affects the hit ratio. If there is only one entry in each set, the cache is call direct-mapped cache. The direct-mapped caches do not have better hit ratio than set-associative caches; for large cache, the cycle time degradation of set-associative design caused by the comparison and selection hardware would outdo the benefit derived from the improved hit ratio [3]. For small caches, increases in size have a much more significant impact on performance than the addition of set associativity.

The interaction of cache access and address translation affects the cache access time and the cache miss service time. Some machines translate virtual address into physical address before accessing the cache, which slows down the cache access. This scheme may increase the machine cycle time or the pipeline slots allocated to memory access. One technique to solve this problem is to do the translation in parallel with the cache access, We use unmapped page offset bits to index the cache. The main disadvantage of this scheme is that the size of cache will be limited by the page size and degree of associativity. For example, a 64 kbytes cache with 4 kbytes page size must be 16 way set-associative. By restricting the mapping of virtual to physical address so that they have the same low order bits of virtual page number and physical frame number, we can solve this problem, but it is too stringent to allocate memory frame efficiently if the cache size is very large. The cache can also be a virtual cache and the translation process is only necessary when cache misses occur. There can be a separate TLB unit between the cache and memory or simply put the TLB into the cache (in-cache address translation mechanism [6]). The drawback in the virtual cache is the synonym problem [2].

The synonym problem occurs when two virtual addresses are mapped to the same physical address. It is the same as the software alias problem. There may be many entries in the caches contains the same data due to the different virtual address. When one of the copies is modified, the other copies must be updated or invalidated to maintain consistency. Similarly, for multiprocessor, multiple private caches may contain many copies of the same data. This causes the cache coherence problem [7]. If we have multiple TLB in the system, we also have the TLB coherence problem since TLB is nothing more than a special virtual address cache.

\section{Problem Statements}

Synonym problem and cache coherence problem are similar. They all involve the multiple copies of the same data. The synonym problem occurs when two different virtual addresses represent the same physical address, but the data coherence problem takes place when different locations of storage contain the data with the same address.

\subsection{Synonym Problem Approaches}

The different virtual addresses of the same physical address make the same data distributed in different sets, or in different entries of the same set. The most expensive solution is adding an invert translation buffer (ITB), a logic circuit that maps physical address back to virtual addresses to check the existence of synonym problem. Note that this mapping is one to many and thus the ITB is complex and not particularly easy to be implemented. Software approaches are more frequent to be selected. Three software methods can be used:

1. one to one mapping: The mapping function of virtual address to physical address is one to one. Since there will not be two different virtual addresses represent the same physical address, there is no synonym problem. The global virtual space of SPUR is this scheme.

2 . handled by system routines of software controlled caches: The caches in the VMP [8-10] are by software controlled. The cache miss, address translation and cache coherence activities are all done by interrupting a CPU and the requests are serviced by software. The synonym problem can be solved in these routines.

3. Restrict synonyms to be equal to module the cache size: Restrict the mapping of virtual pages to physical frames so that all of these virtual addresses have the same low order bits of page number, ie., restrict synonyms to be equal to module the cache size. This is the expansion of the constraint of the paged virtual memory since in the paged virtual memory system all synonyms must be equal to module the page size. Note that the one to one mapping is a special case of this scheme if we consider the size of the cache is infinite. This scheme only works in some virtually addressed caches as will be described in section 2.3 


\subsection{Data Coherence Problem Approaches}

Several algorithms have been proposed for cache coherence in multiprocessor systems. Most of them are applied only to bus based systems called snooping protocols [2,11-19]. In these protocols, each cache monitors the bus transaction to determine the coherence-related operation to act. They are different primarily in how they find out the source of missed block, how they invalidate or update copies and when they update the memory. Due to the bandwidth constraints imposed by a single bus, the scale of this system is limited (probably no more than 20).

Another class of protocols are directory-based [20-26], These protocols keep the coherent information with the main memory modules, instead of distributing them into caches as the snooping protocols. This scheme can support more processors than snooping schemes.

The Software assisted cache coherence schemes[27] detect coherence problem before run-time and insert invalidation instructions in the source code by a compiler. These schemes and the directory-based protocols are suitable for large scale multiprocessor systems with multistage interconnection network.

For the TLB coherence problem, we can handle this problem by implementing a snooping TLB like the snooping cache. But modification of page table is infrequent, it is too expensive to support this unusual operation. We also can implement a special bus operation and TLB snooping is only necessary on this bus transaction. The hardware can thus be reduced. Another simple method is used in our design. we reserve a region in the physical space and the snooping controller considers the transaction to these address as the TLB invalidation commands and no new bus command is required. Partial word or no comparison is necessary to invalidate the correct entries in the corresponding set of the TLB. It only degrades the performance insignificantly. This method only required very little hardware in our design.

\section{Classification of Snooping Caches}

The snooping caches watch all coherence transaction on snooping bus. The interference between the CPU cache access and the bus snooping access is inevitable. This interference can be reduced by using another tag for snooping access, as shown in Figure 1. Based on this basic model shown in Figure 1, we can classify this cache organization by the address used to locate the cache and the type of address in the contents of BTag (used by snooping bus controller to monitor the bus operation) and CTag (used by CPU's cache access). The address can be an unmapped virtual address or a translated physical address. It may be ambiguous to distinguish the differences between physical address and virtual address to index a cache if the size of cache is small, since the frame offset bits of physical address and the page offset bits of virtual address are the same. But we assume that the cache is large enough to make the difference.

In general, based on the above distinction, we have eight combinations and four of them are considered useful:

1) physically addressed physically tagged cache (PAPT cache),

2) virtually addressed virtually tagged cache (VAVT cache),

3) virtually addressed physically tagged cache (VAPT cache),

4) virtually addressed dually tagged cache (VADT cache),

The first three configurations have the symmetric tags, that is, the BTag and CTag contain the same address information. This characteristic makes the chip area to implement the tags less than compared with VADT scheme.

The PAPT scheme as shown in Figure 2.a is a traditional method to parallel the translation processes and cache accesses. It needs a TLB to translate virtual address into physical address and uses the translated address to compare with the physical address contained in the tag field of cache to determine hit/miss condition. The translated physical frame number also combines with the unmapped page offset bits to form the index number of cache. To speed up, the page offset bits are first used to access a line in the cache, then it uses translated physical frame number to select the required word from the line. The TLB must be fast enough in order not to create a critical path in the cache access. This means that TLB must be small and thus is fast enough to compensate the penalty of chip boundary crossing when TLB and cache cannot be integrated in the same chip.

Due to the fastest access time among all cache organizations, The VAVT illustrated in Figure 2.b is suggested recently by many RISC system designs (MIPS-X, SPUR). The cache is directly accessed by virtual address and the hit condition is decided by comparison of virtual tag and the virtual address. Translation is only required when cache miss occurs. Since the hit ratio of large cache is so high that translation processes can be slowed without heavy degradation of performance. Translation can be simply done by software [8] or combining the TLB with cache [6]. It can also use a slow TLB between the cache and memory. The major problem of this scheme is the synonym problem. The one to one address mapping method can solve this problem. Restricting the mapping to equal modulo the cache size only works on direct-mapped cache in uniprocessors. But care must be taken when cache entry replacement takes places, dirty replaced block must be written back first, then we can issue the miss access. Otherwise, the data that are read may be out of date because the up-to-date data are just in the replaced block. This means that the penalty of cache miss is larger and hit ratio will slightly increase. For the set associative caches, the synonym may occur in the same set. The virtual tags fail this method. For multiprocessor, the multiple private caches are logically equivalent to a large set associative cache so this method can not work. The synonym problem also aggravates the complexity of snooping activity. If we only send the physical address in the snooping bus, the destination caches must do an invert translation to map the physical address back into a set of virtual addresses and so they can invalidate or update these copies. SPUR used a global virtual space to prevent the synonym problem. Even so, when cache miss occurs, it may be necessary to send physical address and virtual address at the same time to access the other caches and memory in parallel.

The other major advantage of this scheme is the TLB can be merged into the cache. But data for page protection and statistics, such as page dirty bit, these information must be duplicated in each cache entry. This makes the updating of page table entry (PTE) very difficult. When memory size is large enough, this duplication do not make significant performance degradation [28].

Another drawback of this scheme is that a translation must be performed to get the physical address of the replaced dirty block in order to be written back to memory. This will increase the miss service time and deadlock could occur if the PTE of the replaced block displaces this dirty block. To eliminate this complexity, SPUR keeps physical tag for each block in the tag memory. This increases the tag memory significantly.

A new design scheme called VAPT cache, shown in Figure 2.c is proposed in the MMU/CC design of MARS(see Figure 4). The index used to access cache is derived from virtual address, but the virtual page number is also fed into TLB at the same time. The translated physical address is compared with the cache physical tag to determine hit condition. Since the cache is virtually addressed, as described at the above paragraph, it has the synonym problem. The problem is simply solved by making all of virtual pages mapped to the same physical frame having the same low order bits of virtual page number. We call these bits the cache page number (CPN). The size of CPN is dependent on the cache size. For example, a 64 kbytes direct-mapped cache with 4 kbytes page size, the size of CPN is 
4. In general, if we use $\mathrm{M}$ bits to select a word in the cache and the page size is $2^{* *} \mathrm{~N}$ words, then the size of CPN is M-N. The extra bus signal lines are necessary to carry the CPN information for snooping access to the BTag. These extra lines are limited since the size of CPN is small. For example, a 64 kbytes direct-mapped cache with 4 kbytes page size only needs four lines and 1 Mbytes caches needs eight lines. For this cache, the mapping mapped to the same set but different entries for the set-associative cache has no problem since the tag contains physical addresses. The access speed is the same as VAVT cache and the delay miss technology which informs CPU the hit condition at a later time makes the timing constraint of TLB looser than PAPT cache. Extra signal lines are necessary to maintain cache coherence.

The last class of caches, VADT cache as shown in Figure 2.d, have both physical tag and virtual tag in each entry. In addition to preventing address translation process for the dirty replaced block when cache misses occur, the physical tag also serves as a snooping tag to maintain cache coherence. This can reduce the necessary address information to maintain cache coherence to physical address plus CPN. The advantage of this scheme is the same as the VAVT cache due to the virtual tag. The mapping mapped to different entries in the same set for set-associative cache can be solved but it is more complex than VAPT cache. When cache miss occurs, virtual address is translated into physical address as VAVT does. After that, the physical tag is accessed and compared with the translated physical address to determine whether it is a real miss. The comparison with physical tag can be done in parallel with the memory access. If it is not a real miss, CPU continues execution and the fetched data are discarded.

A brief comparison of different classes of snooping caches is shown in Figure 3 .

\section{Virtual Memory and Caches of MARS}

\subsection{Cache Selection for MARS}

Due to the lack of on-chip data cache for our unique configuration, the need of a fast external cache excludes the use of PAPT cache. As discussed in chapter 2, the three virtually addressed cache organizations have the same access time. We choose the VAPT cache organization because of the following reasons.

1) The constraint of processes sharing is small. The granularity of sharing the two processes is a page. This gives the system better protection. Even though the shared virtual addresses must have the same cache page number, it is easy to match this constraint owing to the large virtual address space.

2) The modification of snooping bus is small. Since the size of cache page number is small, it is easy to be implemented by using the non-used signals of commercial bus.

3) Owing to the physical tag of this scheme, the replaced block can be written back immediately without additional address translation. This simplifies the controller design. For the VAVT cache, a physical tag may be necessary in addition to virtual tag to prevent possible deadlock and reduce the complexity of controller.

4) The protection bits, dirty bit and process identity are kept in TLB without being duplicated for each cache entry. The separation of the TLB and cache makes the total memory cells smaller and increases the probability of integrating cache tag and MMU/CC together. Some states of page such as the dirty bit and access bit are easier to be supported in the TLB than in the cache.

5) The memory cells with two read ports can be used to implement the BTag and CTag. They occupy less silicon area than the two tags with only a single read port of the VADT cache and also simplify the tag updating.

6) If we implement the tags by commercial SRAM's due to the consideration of MMU/CC chip area, the amount of
SRAM's can be reduced. Usually the physical memory is smaller than the physical space. Some high order bits of PPN can be hard-wired to a value and thus save the SRAM's for the tags. For example, if the system has total memory size of 16 Mbytes, only twelve bits of PPN are necessary to be kept in the SRAM's of tags.

In addition to the VAPT scheme, our cache is a write-back cache to reduce the bus traffic and direct-mapped cache to match the cycle time of CPU.

\subsection{Virtual Space of MARS System}

The virtual memory system of MARS is a paged virtual memory with two-level page tables and page size of 4 kbytes. The virtual space of MARS is partitioned into two spaces, user space and system space. The most significant bit, bit 31 , is used to specify the addressed region. User process is assigned to user space and all user processes share the same system space. Each region is given a page table, the system page table (SPT) and the user page table (UPT), and the virtual space for these table are fixed in their virtual space respectively. This decision removes the hardware in the MMU/CC dedicated to the virtual base registers of page table.

The system space is further divided into two regions by the bit 30 , the mapped and unmapped regions. The data of unmapped region are also non-cacheable and the purpose to define this region is to run initializing programs when the system is booted since at this time the contents of page tables, TLB and the caches are all invalid. It also removes the need to design a control bit to bypass the TLB and cache for this period.

Each virtual address has its own page table entry (PTE) and root page table entry (RPTE). The process of generating the PTE and RPTE of a virtual address is very simple. To form the PTE from a virtual address, the most significant bit, i.e. system bit, is reserved and, the other bits are shifted right ten bits and 1 's are inserted. Since the size of PTE is four bytes and aligned on word boundary, the bottom two bits of the address must be " 0 ". Since the RPTE is indeed the PTE's page table entry, the same process is applied to form the RPTE from PTE.

\subsection{Translation Process}

In fact, each access of the cache involves the parallel fetching of external cache for data and the TLB for PTE. The reference of TLB is for access control and address translation. Four events may occur in this process. They are TLB miss, page fault, cache miss and cache hit. TLB miss takes place when the PTE of the address is not in the TLB. Page fault occurs if PTE is in the TLB but this access is illegal. If the access of TLB hits and is permitted but the data are not in the external cache, we call this cache miss. The last and most frequent case is cache hit. In this case, data and their PTE are in the cache and TLB, and the memory operation is allowed to proceed.

The steps of address translaition and cache access are a recursive procedure. At any level of this process, a page fault will abort this activity and exception is notified in the CPU. The exception routine will handle this fault according to the access type and the content of PTE. A TLB miss will make the PTE of the current serviced address be accessed and the depth of this recursive process is increased by one. This recursive call will be terminated when the reference is for the RPTE of the original data address, since the physical address of RPTE has been stored in the RPT base register and this TLB access will be a hit surely. A cache miss will cause the data to be fetched from the memory. This case and the cache hit force recursive process to be returned. If the current access is for PTE or RPTE, the returned data is sent to TLB and one of the TLB entries is displaced. Otherwise, the data will be returned to the CPU.

The PTE and RPTE are not necessary to be cacheable. The cacheability of PTE's can be controlled by the cacheable bit in their PTE. Caching the PTE in the cache will reduce the TLB 
miss service load, but they conflict with the normal data. The cacheable option of PTE enables the OS to trade off this case by the cacheable bit.

\subsection{Snooping bus protocol}

The first step of our project is to build a multiprocessor workstation with 6 to 12 processors. The snooping protocol (see Figure 5) is well suitable to this size of processor number. Two major classes of snooping protocol are the write-invalidate and the write-broadcast protocols. Both techniques have been criticized for being unable to achieve good bus performance across all cache configurations[37]. We select the write-invalidate because it is simpler to be implemented and the test-and-set synchronization operation can be performed by the local cache write operation, which simplifies the bus design.

In addition, we support the local access to the distributed, interleaved global memory in each CPU board. One bit in the page table entry is used to set the corresponding page as a local page and the access is directed to the on-board memory without passing through the bus.

\subsection{Simulation}

In a shared-bus multiprocessor system, the bus traffic is the bottleneck of the overall system performance. To boost the overall system performance, we must minimize the bus requirement of each individual processor. For cache based multiprocessor systems, a good cache coherence protocol can further reduce the bus request. In order to demonstrate how efficient the coherence protocol is, we use a simulation model[39] to perform system performance evaluation. We select the appropriate parameters value for RISC architecture. The reference stream of each processor is viewed as the merging of two reference streams: one being the reference to shared blocks and the other being the reference to private block. Each time a memory reference is called for.

The processor generates a reference to a shared block with the probability SHD and a reference to a private block is generated with the probability (1-SHD). The shared data are specified by a block number and the private data are processed by probability. PMEH is the hit ratio of the on-board memory. When cache miss occurs, either for shared block or for a private block, a block must be ejected to make room for the new block. If the selected block is private, it is modified and needs to be written back with probability MD. Figure 6 shows the simulation parameters and their corresponding range[36].

Our cache coherence protocol is similar to the Berkeley's except two local states. We compare our protocol with Berkeley's by using the same simulation parameters. The output of the simulation includes processor utilization and bus utilization. From Figure 7 to Figure 12,we change PMEH value from 0.1 to 0.9 to observe the effect of the memory allocation. Figure 7\&8 give the processor and bus utilization improvement percentage of MARS when a write buffer is placed between the cache and bus. When system is composed of 10 processors, adding write buffer can increase the performance by $15 \sim 23 \%$. Figure $9 \& 10$ show the processor utilization improvemen percentage and Figure $11 \& 12$ show the bus utilization improvement percentage compared with that of Berkeley with/without a write buffer. When write buffer is adopted, the maximum improvement can reach $142 \%$. From the simulation results, we believe that the cache coherence protocol of MARS can achieve a better performance by interleaving the global memory and adopting a write buffer.

\section{Implementation of MMU/CC}

\subsection{Data path of MMU/CC}

The block diagram of MMU/CC is shown in Figure 13. The TLB module is a two-way virtually addressed virtually tagged cache with 128 entries. In this module, TLB_RAM is a
RAM with 65 words. The first 64 words contain the 64 sets of the TLB and the first come $(\mathrm{Fc})$ bits for each set. The Fc bit selects the entry which first enters the TLB to be the victim when there is no room for the new coming PTE. The use of FIFO replacement algorithm instead of LRU also reduce the hardware and the cycle time of TLB because the LRU algorithm needs a read-and-modify operation for each TLB access. The 65th set is used to put the physical Base Registers (RPTBR) of the URPT and SRPT and should be properly set by the OS during the context switching. This design reduces the need to insert these base registers into the other datapath and the multiplexer to read these registers. The load/store of these two registers are also simplified. Another advantage is that the reference of RPTBR is the same as the PTE reference of TLB except that the MSB of the TLB RAM's address is set to " 1 ". This characteristic makes the implementation of the recursive address translation algorithm in very easy by just changing the MSB of TLB_RAM's address without impacting the time to compare the PPN's of TLB and external tag. Though this design may increase the decoding time because of the additional input to the decoder, this signal always arrives before the other inputs and it has little impact on the cycle time.

The VTag_DP, PID_DP, State_DP and TLB_PPN_DP have the similar structures. They control the $I / O$ of the two addressed TLB entries and determine the hit conditions of TLB and external caches by the comparators. The bits of the two entries of TLB are interleaved in the TLB_RAM and these parallel datapaths process two bits of the same set in each bit slice. This design is chosen because the size of two bit slices of TLB RAM well matches the size of one bit slice of these parallel datapaths. The Access_Check module is a group of random logic to check the illegal access for protection or the write to a clean page by dirty bit. The updating of page dirty bit is not implemented by hardware because the probability of occurrence is low and the write to PTE involves the coherent problem.

The PPN DP is the datapath used to process the higher order bits of physical addresses for memory access and snooping access. The Vadr_DP generates the virtual address and the PTE/RPTE. When page fault occurs, the Bad_adr_ $\Phi 1$ is used to latch the virtual address which the CPU sends out. The Bad_adr_ $\$ 1$ does not latch the PTE/RPTE address when page fault happens during the access of PTE. This is to reduce the need for hardware. The exception code of MMU/CC is used to carry this information for software approaches. The shifter10/20 module shifts the virtual address and inserts " 1 " to form the virtual address of the PTE and RPTE. In fact, this module is implemented by routing. Due to the fixed virtual space for UPT/SPT, the address generation of PTE/RPTE is simplified to only the multiplexers and the shifter routing. The Cindex_DP processes the higher order bits of virtual addresses for memory access and snooping access.

\subsection{Controller of the MMU/CC}

The block diagram of controllers of $\mathrm{MMU} / \mathrm{CC}$ is shown in the Figure 14. The CPU cache access controller (CCAC) controls the command of the CPU and requests the memory access controller (MAC) if memory access is necessary. The main jobs of MAC are to write out a dir $\$$ block, request the bus controller, update the BTag and read in the missed block. MAC can be further partitioned into two sub-controllers, i.e. the data controller (MAC_DC) and address controller (MAC_AC). The MAC_DC controls the data to/from the cache for memory read/write and updates the CTag. The MAC_AC sends out the address of memory access and updates the BTag. Two controllers are used for the snooping access, the Snooping BTag controller (SBTC) and Snooping CTag controller (SCTC). The SBTC accepts the snooping command from bus controller and accesses BTag to determine the snooping hit condition. If necessary, it update the state of BTag and requests the SCTC, 
otherwise it waits for the next snooping command. The SCTC is used to update CTag and access the cache data.

\subsection{Result}

The layout of MMU/CC, as Figure 15 shows, has been generated by GENESIL. The used technique adopted here is a double-metal single-poly $1.2-\mu \mathrm{m} n$-well CMOS process. The chip integrates 68861 transistors on a die measuring 7.77 $\mathrm{mm} * 8.81 \mathrm{~mm}$ and consumes $1.2 \mathrm{~W}$. The pin number is 184 and 38 pins for power. The chip size is bounded by the bounding pads. We have been modifying the external interface to make the pin number less than 144 and integrating more functions into this chip.

\section{Conclusion}

In this paper, we classify and compare different cache organizations based on the type of address in the tags. For their fast access time, the virtual addressed caches are the new trend for cache design. The VAVT and VADT caches can move the TLB into data caches and discards the TLB coherent problem. For the VAPT cache, the software constraint to solve the synonym problem, bus signal lines, control complexity and memory cells necessary to implement the tags are smaller than those of the VAVT and VADT caches. A TLB is necessary and the synonym problem is solved by restricting the mapping to be equal modulo cache size.

The MMU/CC is implemented by GENESIL due to its fast turnaround time. By implementing the page table base registers into the TLB, the recursive translation algorithm is easy to achieve and the timing of critical path is looser. The cacheable/non-cacheable option of the translation algorithm makes the OS easy to trade off the TLB miss service time and contentions between the PTE and data in the cache according to the characteristic of programs. The virtual spaces of page tables are also fixed to reduce hardware significantly. The snooping protocol of cache coherency is write-invalidate protocol with supporting local memory to reduce bus traffic. It is easy to modify this protocol based on the future bus design and application without changing the basic structure.

\section{REFERENCES}

1. A. J. Smith, "Cache Memories," ACM Computing surveys, Vol.14, No.3, Sept. 1982, pp.473-530.

2. James R. Goodman, "Using Cache Memory To Reduce Processor-Memory Traffic," Proc. 10th Symposium on Computer Architecture, 1983, pp.124-131.

3. Steven Przybylski, Mark Horowitz, John Hennessy, "Performance Tradeoffs in Cache Design," Proc. 15th Symposium on Computer Architecture, 1988, pp.290-298.

4. J. H. Chang, H. Chao, and K. So, "Cache Design of A Sub-Micron CMOS System/370," Proc. 14th Symposium on Computer Architecture, 1987, pp.208-213.

5. Kai Hwang and Faye' A. Briggs, "Computer Architecture and Parallel Processing," 1984, pp.60-80.

6. Wood, D. A. et al., "An In-cache Address Translation Mechanism," Proc. 13th Symposium on Computer Architecture, June 1986, pp.358-365.

7. L. M. Censier and P. Feautrier, "A New Solution to Coherence Problems in Multicache Systems," IEEE Transactions on Computers, Vol. C-27, No.12, December 1978.

8. D. R. Cheriton, G. Slavenburg, and P. Boyle, "Software controlled caches in the VMP multiprocessor," 13 th Symposium on Computer Architecture, June 1986, pp.366-374.

9. D. R. Cheriton, A. Gupta, P. Boyle, and H. A. Goosen,

"The VMP multiprocessor: Initial experience, refinemens and performance evalution," 15 th Symposium on Computer
Architecture, June 1988, pp.410-421.

10. D. R. Cheriton, H. A. Goosen, and P. D. Boyle, "Multi-level Shared Caching Techniques for Scalability in VMP-MC," 16th Symposium on Computer Architecture, June 1989, pp.16-24.

11. Charles P. Thacker and Lawerence C. Stewart, "Firefly: a Multiprocessor workstation," Proc. of ASPLOS II, October 1987, pp.164-172.

12. Frank S. J. "Tightly Coupled Multiprocessor System Speed Up Memory Access Time," Electronics, January 1985.

13. Larry Rudolph, Zary Segal, "Dynamic Decentrialized Cache Schemes for MIMD Parallel Processors," 11 th Symposium on Computer Architecture, June 1984, pp.340-347.

14. Mark S. Papamacros and Janak H. Patel, "A Low-overhead Coherence Solution for Multiprocessor with Private Cache Memories," 11 th Symposium on Computer Architecture, June 1984, pp.348-354.

15. R. H. Katz, S. J. Eggers, D.A. Wood, C. L. Perkins, R. G. Sheldon, "Implementing A Cache Consistency Protocol," 12 th Symposium on Computer Architecture, June 1985, pp. 276-283.

16. Paul Sweazey and Alan Jay Smith, "A Class of Compatible Cache Coherency Protocols and Their Support by the IEEE Futurebus," 13th Symposium on Computer Architecture, June 1986, pp.414-423.

17. Philip Bitar and Alvin M. Despain, "Multiprocessor Cache Synchronization Issues, Innovation, Evolution," 13th Symposium on Computer Architecture, June 1986, pp.424-433

18. Andrew W. Wilson Jr., "Hierarchical Cache/Bus Architecture for Shared Memory Multiprocessors," 14th Symposium on Computer Architecture, June 1987, pp.244-252. 19. Atsuhiro Goto, Akira Matsumoto, Evan Tick, "Design and Performace of a Coherent Cache for Parallel Logic Programming Architecture," 16th Symposium on Computer Architecture, June 1989 , pp.25-33.

20. C. K. Tang, "Cache Design in the Tightly Coupled Multiprocessor system," AFIPS Conference Proc., National Computer Conference, June 1976, pp.749-753.

21. Lucien M. Censier and Paul Feautrier, "A New Solution to Coherence Problem in Multicache Systems," IEEE Transactions on Computers, December 1978, pp.1112-1118.

22. James Archibald and Jean-Loup Baer, "An Economical Solution to the Cache Coherence Problem," 11 th Symposium on Computer Architecture, June 1984, pp.355-362.

23. Wei C. Yen, David W. L. Yen, and King-Sun Fu.,"Data Coherence Problem in a Multicache System," IEEE Transactions on Computers, January 1985, pp.56-65.

24. Christoph Scheurich and Michel Dubois, "Corect Memory Operation of Cache-based Multiprocessor," 14th Symposium on Computer Architecture, June 1987, pp.234-243.

25. Anant Agarwal, Richard Simoni, John Hennessy, and Mark Horowitz, "An Evaluation of Directory Schemes for Cache Coherence," 15th Symposium on Computer Architecture, June 1988 , pp.280-289.

26. Per Stenstrom, "A Cache Consistency Protocol for Multiprocessors with Multistage Networks," 16th Symposium on Computer Architecture, June 1989, pp.407-415.

27. Hoichi Cheong and Alexander V. Veidenbaum, "A Cache Coherence Scheme With Fast Selective Invalidation," 15th Symposium on Computer Architecture, June 1988, pp.299-307. 28. David A. Wood and Randy H. Katz, "Supporting Reference and Dirty Bits in SPUR's Virtual Address Cache," 16th Symposium on Computer Architecture, June 1989, pp.122-129. 29. G. S. Jang, F. Lai, H. C. Lee, Y. C. Maa, T. M. Parng, and J. Y. Tsai, "MARS - Multiprocessor Architecture Reconciling Symbolic with numerical Processing, "International Symposium on VLSI Technology, System, and Applications, May $17-19,1989$.

30. Y.-C. Maa, "Designing Instruction Fetch Unit for the 
MARS System," Master Thesis, Computer Science Div., Dept. of Electrical Engineering, National Taiwan University, June. 1989.

31. W.-C. Tsai, "The Design and Implementation of Instruction Fetch Unit of the MARS System," Master Thesis, Computer Science Div., Dept. of Electrical Engineering, National Taiwan University, June. 1990

32. G.-S. Jang, "The Design of Integer Processing Unit for the MARS Syatem," Master Thesis, Computer Science Div., Dept. of Electrical Engineering, National Taiwan University, June. 1989.

33. C.-J. Horng, "The Design and Implementation of Integer Processing Unit (IPU) for the MARS Syatem," Master Thesis, Computer Science Div., Dept. of Electrical Engineering, National Taiwan University, June. 1990.

34. J.-Y. Tsai, "The Design of List Processing Unit for the MARS Syatem," Master Thesis, Computer Science Div., Dept. of Electrical Engineering, National Taiwan University, June. 1989

35. C.-Y. Chen, "The Design and Implementation of List Processing Unit (LPU) for the MARS Syatem," Master Thesis,

Computer Science Div., Dept. of Electrical Engineering, National Taiwan University, June. 1990.

36. L.-M. Tzeng, "MARS Performace Evaluation with Different Interconnection Networks," Master Thesis, Computer Science Div., Dept. of Electrical Engineering, National Taiwan University, June. 1989

37. C. Scheurich and M. Dubois, "The Design of a Lockup-Free Cache for High-Performance Multiprocessors," Proc. Supercomputing'88, November, 1988, pp.352-359.

38. S. J. Eggers and R.H. Katz, "Evaluating the Performance for Four Snooping Cache Coherency Protocols," 16th Symposium on Computer Architecture, June 1989, pp.2-15. 39. J. Archibald, J. L. Bear, "Cache Coherence Protocals Evaluation Using a Multiprocessor simulation model," ACM trans. Computer, vol. 4, No. 4, Nov. 1986, pp. 273 298.

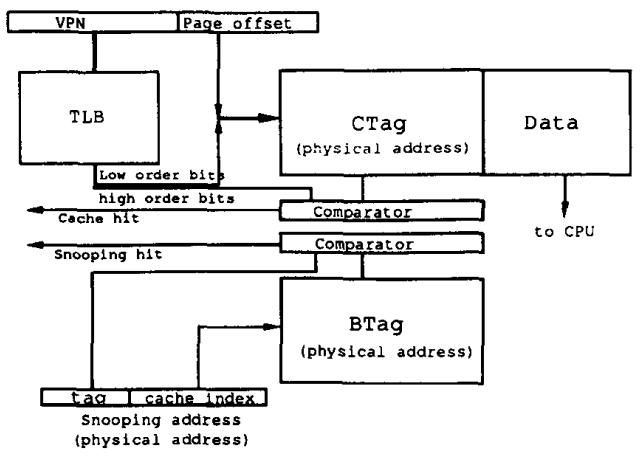

Figure 2.a. Physically addressed physically tagged cache

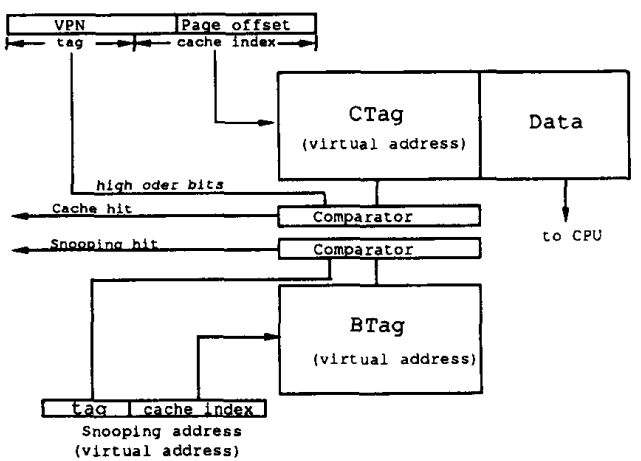

Figure 2.b. Virtually addressed virtually tagged cache

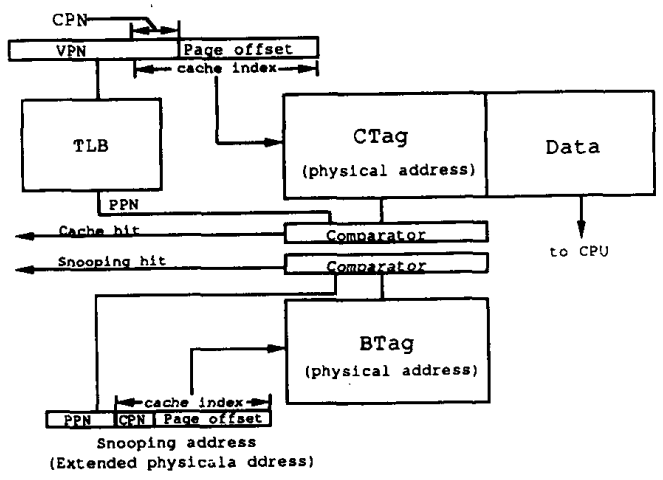

Figure 2.c. Virtually addressed physically tagged cacine

Figure 1. Snooping cache model 


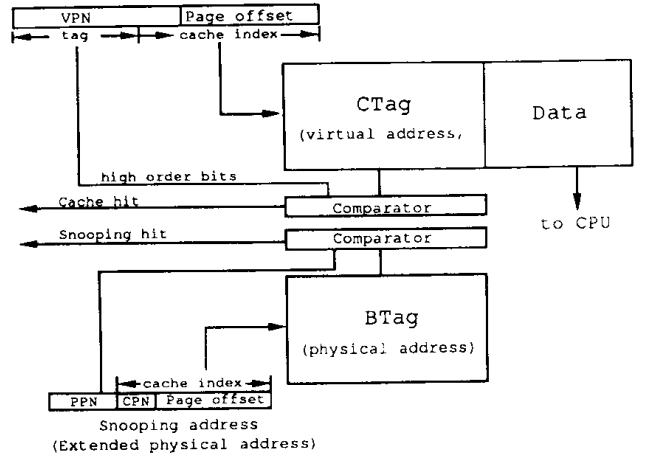

Fig 2.d. Virtually addressed dually tagged cache
The requesting cache

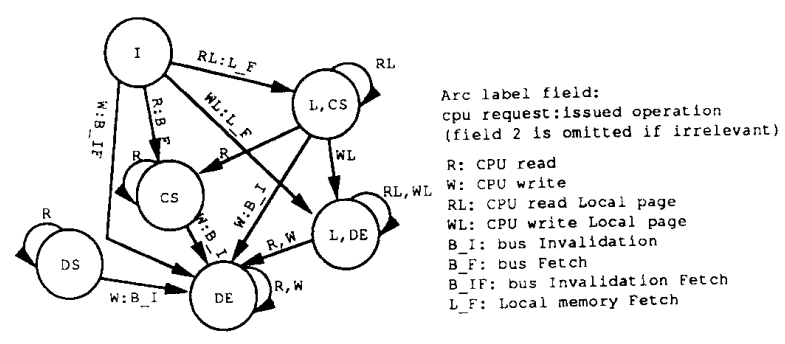

The other caches

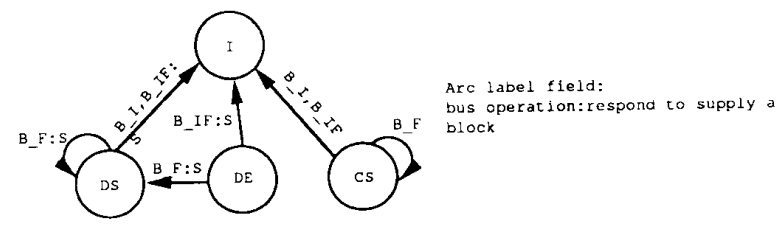

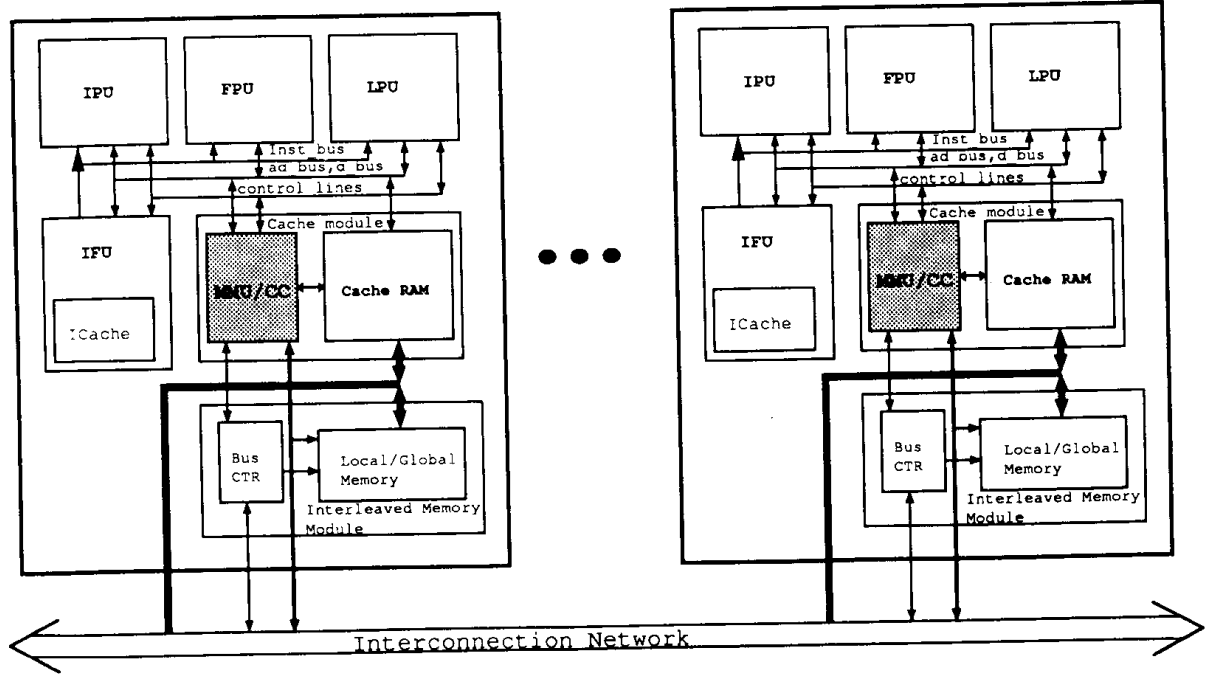

Figure 4. Interboard Architecure of the MARS System 


\begin{tabular}{|c|c|c|c|c|}
\hline $\begin{array}{lll} & \text { caches } \\
\text { issue } & \\
\end{array}$ & PAPT & VAVT & VAPT & VADT \\
\hline cache access speed & slow & fast & fast & fast \\
\hline have synonym problem? & no & yes & yos & yes \\
\hline $\begin{array}{l}\text { synonym i: wiem can be } \\
\text { solved by global viftual space }\end{array}$ & * & yes & yes & yes \\
\hline $\begin{array}{l}\text { can be solved by } \\
\text { equal modulo the cache size }\end{array}$ & * & no & yes & yes \\
\hline Need TLB ? & yes & option & yes & option \\
\hline TLB speed requi rement & high speed & Low speed & average speed & Low speed \\
\hline TLB coherence problem ? & yes & - & yes & * \\
\hline symmetric tags & yes & yes & yes & no \\
\hline Number of memory celis in TLB & $50 * 128$ & 0 & $50 * 128$ & 0 \\
\hline $\begin{array}{l}\text { Number of memory cells in cache } \\
\text { tags } \\
\text { include the physical tag) } \\
\text { a: cells with two read ports } \\
\text { b: cells with one read port }\end{array}$ & $\begin{array}{c}17 * 4 k * a \\
(17 * 4 k * a)\end{array}$ & $\begin{array}{l}23 * 4 k * a+ \\
3 * 4 k * b \\
(23 * 4 k * a+ \\
23 * 4 k * b)\end{array}$ & $\begin{array}{c}22 * 4 k * a \\
(22 * 4 k * a)\end{array}$ & $\begin{array}{c}(26+22) * 4 k * b \\
(46 * 4 k * b)\end{array}$ \\
\hline $\begin{array}{l}\text { number of bus address lines for } \\
\text { maintaining cache coherence } \\
\text { (and access memory in parallel) }\end{array}$ & $\begin{array}{c}32 \\
(32)\end{array}$ & $\begin{array}{l}38 \\
(58)\end{array}$ & $\begin{array}{l}37 \\
(37)\end{array}$ & $\begin{array}{l}37 \\
\text { (37) }\end{array}$ \\
\hline $\begin{array}{l}\text { granularity of protection } \\
\text { and sharing }\end{array}$ & $\begin{array}{l}\text { 4k byces } \\
\text { (a page) }\end{array}$ & $\begin{array}{r}1 \text { glga bytes } \\
\text { (a segment) }\end{array}$ & $\begin{array}{l}4 k \text { bytes } \\
\text { (a page) }\end{array}$ & $\begin{array}{l}1 \text { giga bytes } \\
\text { (a segment) }\end{array}$ \\
\hline $\begin{array}{l}\text { NOTE: The size of virtual and phys } \\
\text { The VAPT cache are assumed to be } \\
\text { I2g kbytes direct-mapped cache, } \\
\text { each tag. also a segement size of } \\
\text { For caches with TLB, the TLB is as }\end{array}$ & $\begin{array}{l}1 \text {. Address are } \\
\text { same as the } \\
\text { serent states, } \\
\text { iga bytes tor }\end{array}$ & sumed to be 32 & $\begin{array}{l}\text { bits. } \\
\text { a bits global vit } \\
\text { its and one page } \\
\text { g. }\end{array}$ & $\begin{array}{l}\text { irtual space, } \\
\text { dirty bit in } \\
\text { iles. }\end{array}$ \\
\hline
\end{tabular}

Summary of Simulation Parameters
\begin{tabular}{|c|c|c|c|}
\hline Data cache hit ratio & \multicolumn{2}{|c|}{$97 \%$} \\
\hline Pipcline cycle & \multicolumn{2}{|c|}{$50 \mathrm{~ns}$} \\
\hline \multicolumn{2}{|c|}{ Bus cycle } & \multicolumn{2}{|c|}{$100 \mathrm{~ns}$} \\
\hline Memory cycle & \multicolumn{2}{|c|}{$200 \mathrm{~ns}$} \\
\hline Data cache size & \multicolumn{2}{|c|}{$256 \mathrm{k}$ bytes } \\
\hline \multicolumn{2}{|c|}{ SHD } & \multicolumn{2}{|c|}{$0.1 \% \sim 5 \%$} \\
\hline MD & $30 \%$ & LDP & $21 \%$ \\
\hline PMEII & $40 \%$ & STP & $12 \%$ \\
\hline
\end{tabular}

LDP : probability of loed

STP : probability of store

SFD : probability of reference to shared data

MD : probability of writing back privale data is modified

PMEH : local memory hit retio

Figure 6. Summary of simulation parameters

Figure 3. Comparison of snooping caches
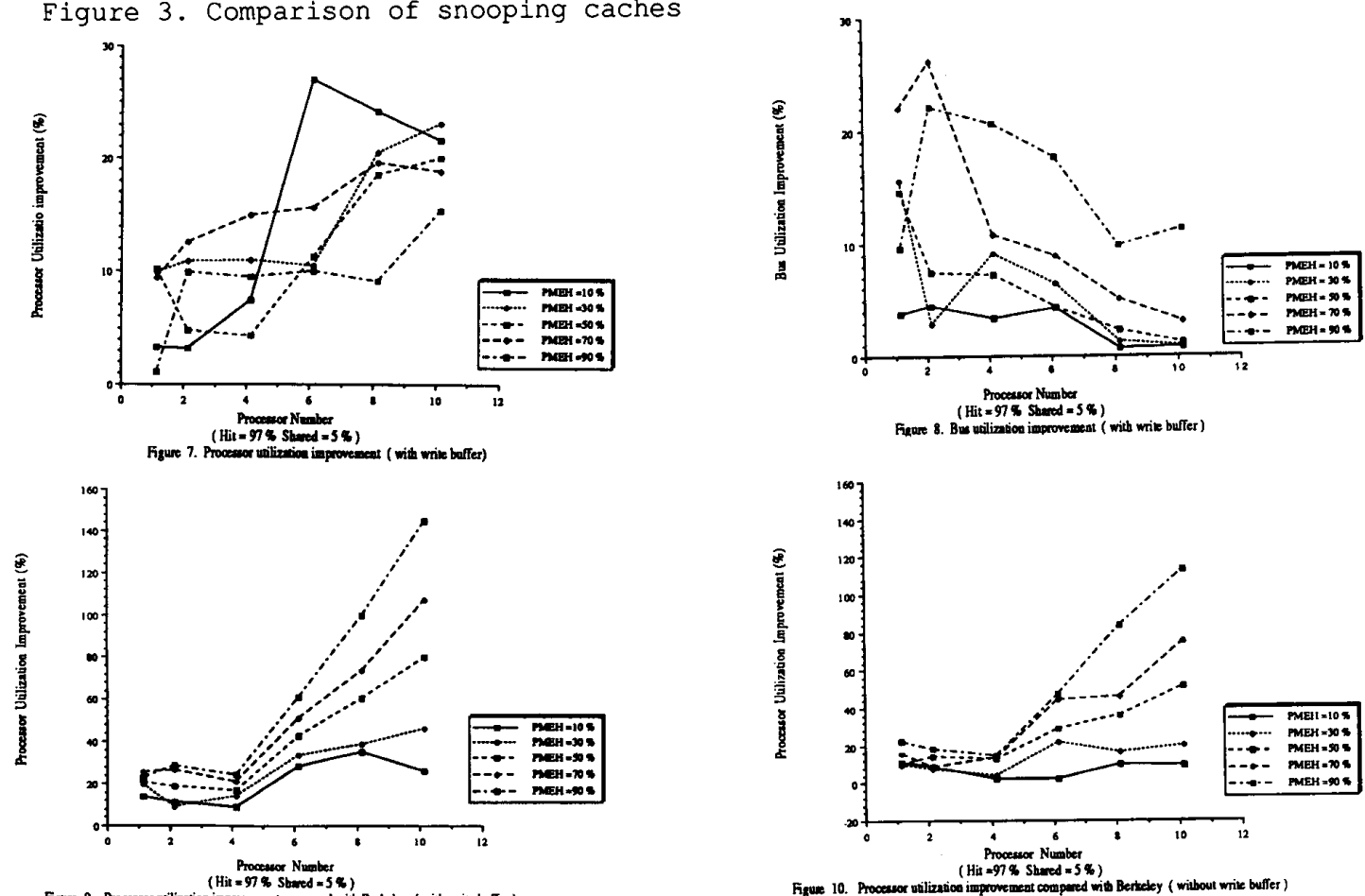

207 

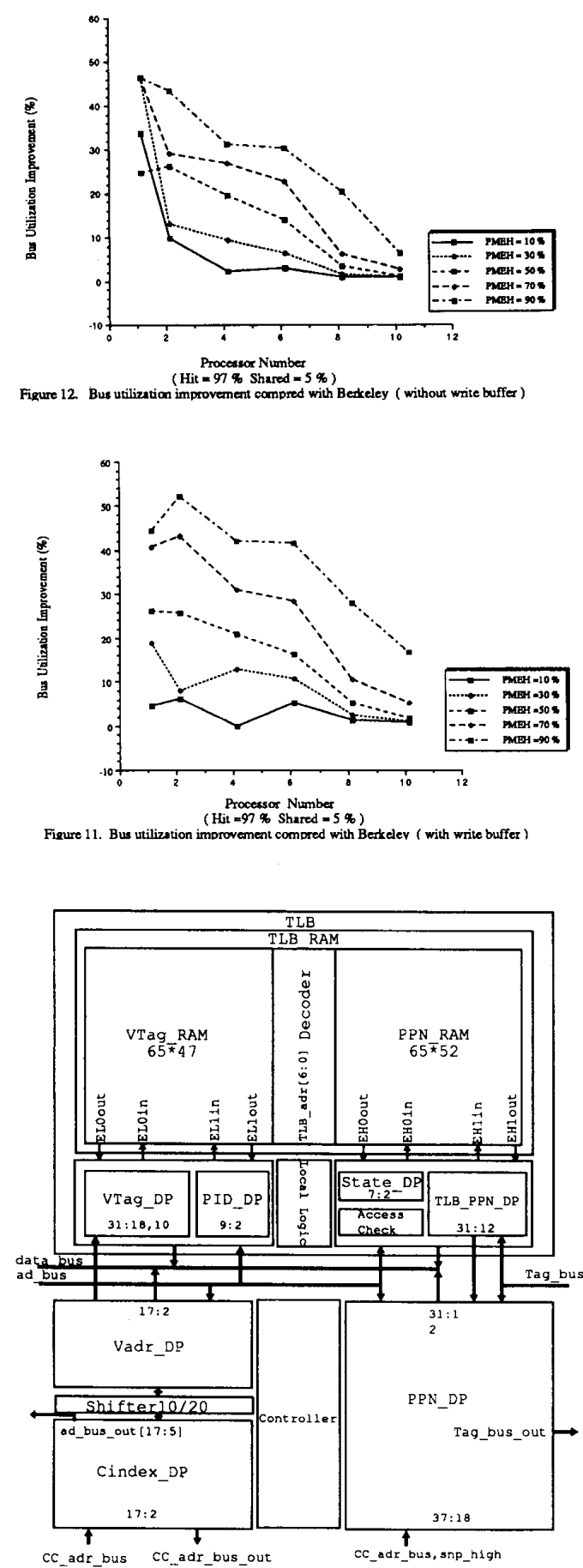

Figure 13. The block diagram of $\mathrm{MMU} / \mathrm{CC}$

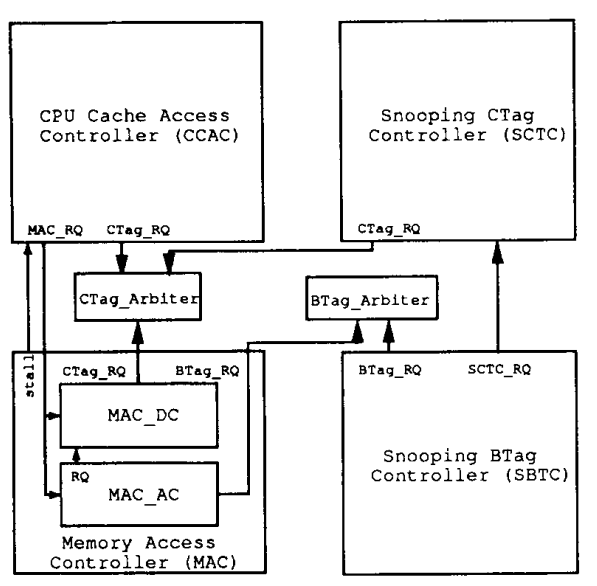

Figure 14. The controllers of MMU/CC

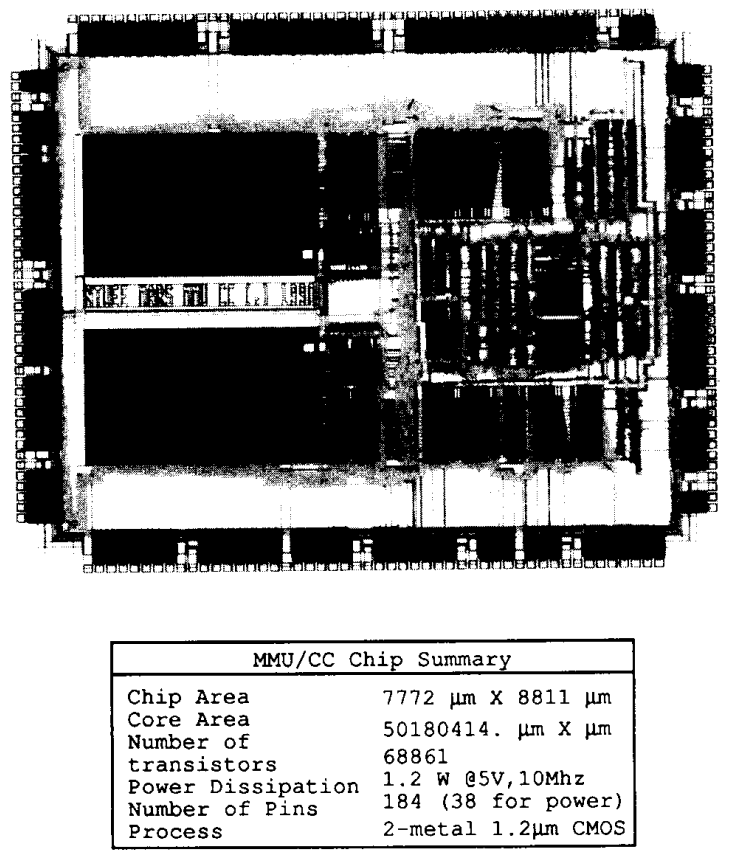

Figure 15. Layout of MMU/CC 